\title{
Nanomaterial-based environmental sensing platforms using state-of-the-art electroanalytical strategies
}

\author{
Wei $\operatorname{Jin}^{1 *}$ and Govindhan Maduraiveeran ${ }^{2 *}$
}

\begin{abstract}
Electroanalytical techniques have been extensively employed in the advancement of sensor platforms based on nanomaterials owing to their rapid response, high sensitivity, and selectivity. It is of immense significance for the swift and sensitive detection of environmental pollutants or contaminants such as a major group of unregulated chemicals such as heavy metals, inorganic anions, phenolic compounds, pesticides, and chemical warfare reagents, which may cumulatively resource severe harm to human health and environmental. These environmental pollutants are regularly obtained from a large group of unmaintained compounds/complexes, containing industry, human, and animal fecal waste; natural toxins; drinking water disinfection by-products; personal care products; pharmaceuticals; food materials through food preparation and packaging processes, etc. The present minireview will display various concepts and advancements of electroanalytical techniques and their potential applications in environmental sensing. The introduction of novel electroanalytical tools and nanostructured electrode surfaces may demonstrate even higher sensitive and selective sensor platforms. Electroanalytical methods possess passionate importance in the analytical research community, and they serve as ideal tactics, which display several features such as rapid response, robustness, high selectivity and sensitivity, cost-effective miniaturization, and the perspective for online monitoring towards environmental, food, and biomedical applications. The advancement and prospects for the applications of electroanalytical techniques using nanomaterials in the design of environmental sensor platforms will also be discussed.
\end{abstract}

Keywords: Environmental monitoring, Electrochemical methods, Sensor platforms, Pollutants, Nanomaterials

\section{Introduction}

Electroanalytical techniques are usually the inter-conversion between electricity and chemistry, which measure the electrical response of current, potential, or charge with respect to the variation of chemical reactions/parameters (Wang 2002). One main advantage of electrochemical sensing is the direct analysis of analyte information in the compact, simple, and portable electrochemical setup. General electrochemical cell for environmental detection mainly consists of an ionic conductor (electrolyte) and an electronic conductor (electrode) (Bansod et al. 2017). The electrochemical sensing reactions occur at the interface between the reactant-bearing

\footnotetext{
* Correspondence: wjin@ipe.ac.cn; maduraiveeran.g@ktr.srmuniv.ac.in ${ }^{1}$ National Engineering Laboratory for Hydrometallurgical Cleaner Production Technology, Institute of Process Engineering, Chinese Academy of Sciences, Beijing 100190, China

${ }^{2}$ Department of Chemistry, Research Institute, SRM University, Chennai, Tamil
} Nadu 603203, India

electrolyte and the working electrode (WE), in which the potential is obtained with respect to the reference electrode $(\mathrm{RE})$ and the current is measured with respect to counter electrode (CE), respectively. In order to provide electrical excitation and receive the sensing response, these electrodes are connected to a laboratory or in-field portable electrochemical workstation embedded with a required power source as shown in Fig. 1. And the workstation is linked to a computer installed with corresponding software to interpret and analyze the sensing data. It should be noted that the two-electrode cell is also available for the environmental sensing, depending on the target electrical signal and corresponding electrochemical techniques. Analytical electrochemistry is the application of electrochemical processes on the systems to measure the quantity of a species of interest, and it is mainly concerned with the measurements of electrical quantities of current, potential, charge, and their 


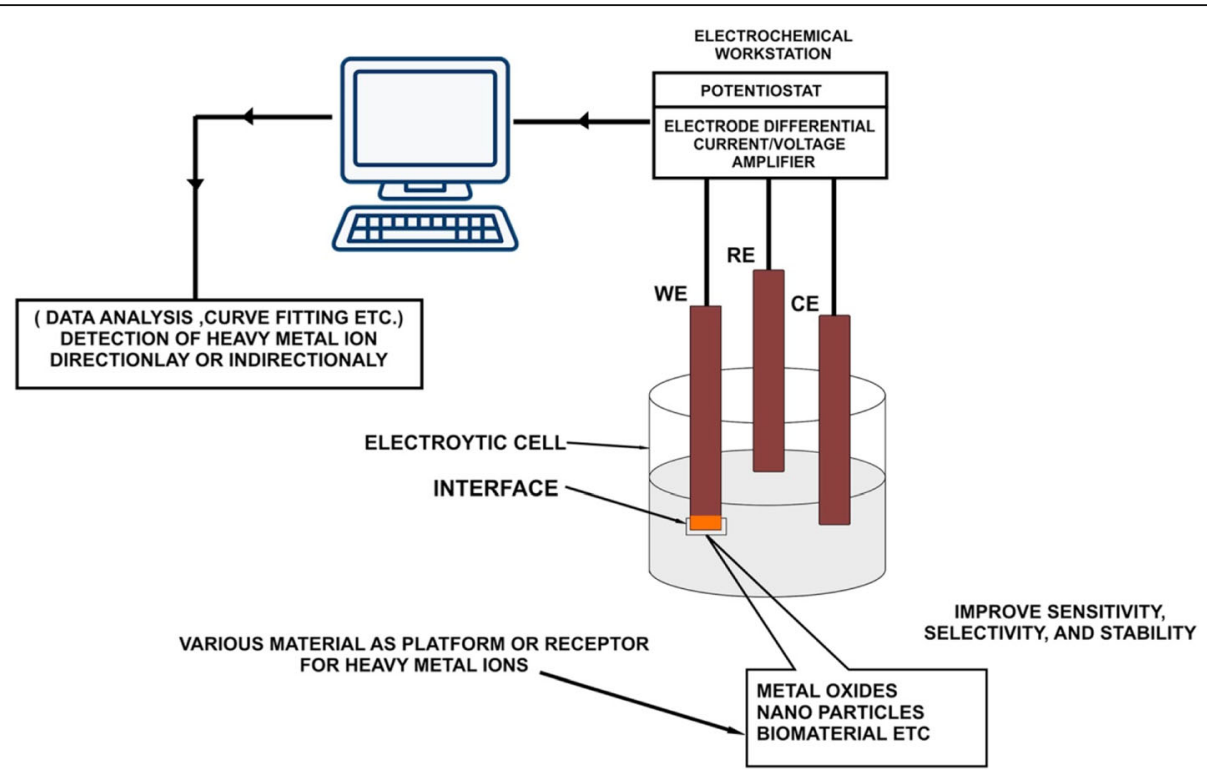

Fig. 1 General setup for electrochemical cell (reprinted with permission from Reference Bansod et al. 2017). ๑ 2017 Elsevier

chemical parameters. Electroanalytical chemistry is much fascinated, and it is lengthened to new phases of environment and medicine by the design of tailored electrochemical interfaces, molecular monolayer structures, the coupling of electrochemical transducers with nanoscale materials and chemical/biological components, and the development of analytical techniques.

The type of quantitative electrical signal originates from many different electroanalytical methods, leading to a wide range of environmental monitoring applications based on nanomaterials (Shao et al. 2010). For the pollutant detection (Jin and Maduraiveeran 2017; Maduraiveeran and Jin 2017), electrochemical techniques are mainly classified into potentiometric, potentiostatic, electrochemical impedance spectroscopy (EIS), conductometric, and electrochemiluminescent methods. In most of these methods, either current or potential is controlled to determine the change of another parameter. However, there are also some techniques in which no control excitation is provided (at zero current), while equilibrium potential is obtained at an ion-selective membrane (Qin et al. 2002). As illustrated in Fig. 2, there are three kinds of environmental gas sensors, including potentiometric sensor for voltage measurement, amperometric sensor for current measurement, and conductometric sensor for conductivity measurement ( $\mathrm{Li}$ et al. 2017). Therefore, the specific electrochemical techniques with corresponding recent advances are described in the following sections.

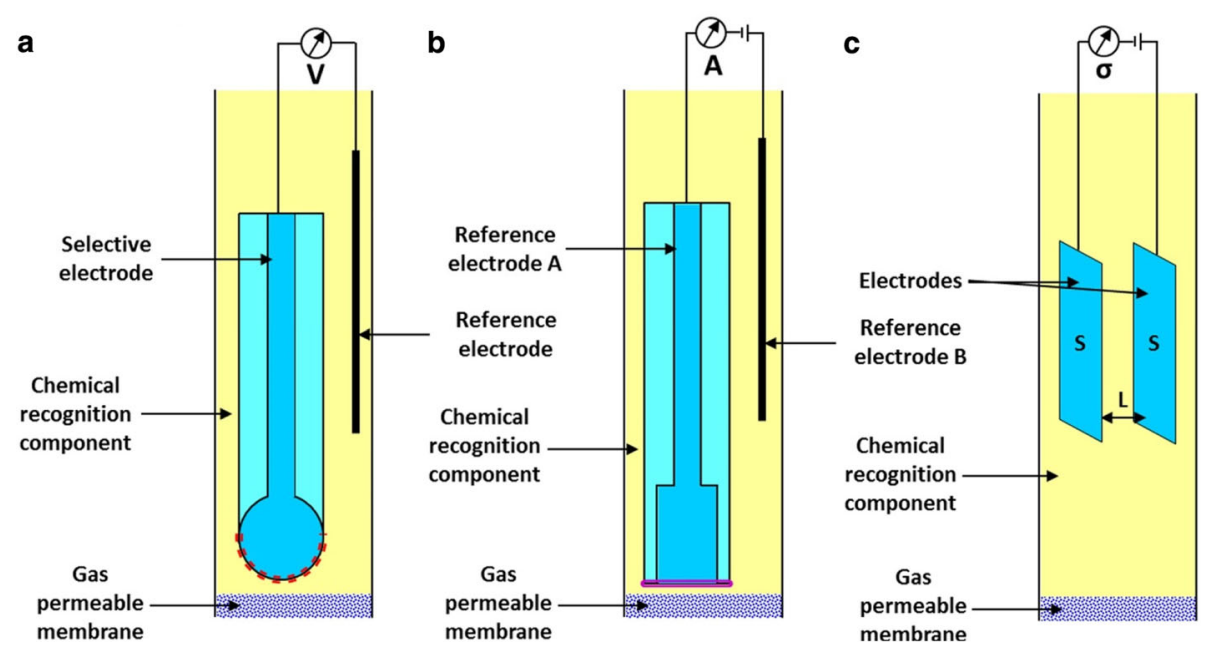

Fig. 2 Schematic diagrams of three typical membrane-based electrochemical gas sensors. a Potentiometric sensor. b Amperometric sensor. c Conductometric sensor. (Reprinted with permission from Reference Li et al. 2017). ๑ 2017 Elsevier 


\section{Review}

\section{Potentiometry}

Potentiometry is a static (zero-current) method in which the target analyte value is measured by the potential generated across an ion-selective electrode (ISE) (Karami et al. 2003). It is an interesting area of electroanalytical research owing to high accuracy, quick response, nondestructive, and cost-effective analysis (Matzeu et al. 2015). The glass electrode employed to determine the solution $\mathrm{pH}$ is the most common example of ISE. The response of an ion-selective electrode is given by Eq. 1 as follows:

$$
E=E o+\frac{R T}{z F} \ln a
$$

in which $E$ is the measured potential (in volts), $E_{0}$ is a characteristic constant for the ISE system, $R$ is the gas constant, $T$ is the absolute temperature, $z$ is the signed ionic charge, $F$ is the Faraday constant, and $a$ is the activity of analyte. ISEs incorporate a selective polymeric membrane to decrease the matrix interferences; thus, ideally, it responds only to one target analyte (Dimeski et al. 2010). Strictly, the potential is obtained as a function of analyte activity rather than concentration. It has been widely employed for the pollution monitoring, such as $\mathrm{CN}, \mathrm{F}, \mathrm{S}, \mathrm{Cl}$, and $\mathrm{NO}_{3}$ in industrial effluents, natural waters, and agriculture processing (Crespo 2017; Zuliani and Diamond 2012). As illustrated in Fig. 3 (Karami et al. 2003), the $\mathrm{Pb}^{2+}$ concentration was successfully detected using the flow injection potentiometry at a new coated graphite ionselective electrode.

A recent development of ion-selective electrodes is ion-selective field-effect transistors (ISFETs) and is displayed in Fig. 4 (Sudibya et al. 2011; Chin et al. 2001). The sensing signal is depending on the interaction or absorption of a charged species, with the interface of FET. Subsequently, the surface potential is changed, and thus, the current flow in the conductive channel of FET is affected via the field effect. In order to selectively detect particular species and obtain an electrical "fingerprint," the FET surface is usually functionalized with corresponding binding groups. The main superiority of such devices is the ultra-small size (below $0.1 \mathrm{~mm}^{2}$ ), which allows low-cost mass-produced preparation with integrated circuit technology.

\section{Potentiostatic techniques}

Potentiostatic techniques (Ricci et al. 2007) involve the use of a potentiostat instrument to control the potential and deal with the charge transfer processes at the electrode/ electrolyte interface. As a result, they can determine the concentration of many environmental species including the electroactive compounds from electrochemical reduction or oxidation and the non-electroactive compounds from indirect or derivatization electrochemical procedures (Jin et al. 2016; Jin et al. 2010). In comparison with potentiometry, the advantages of potentiostatic techniques are high sensitivity and selectivity, wide linear range, portable and

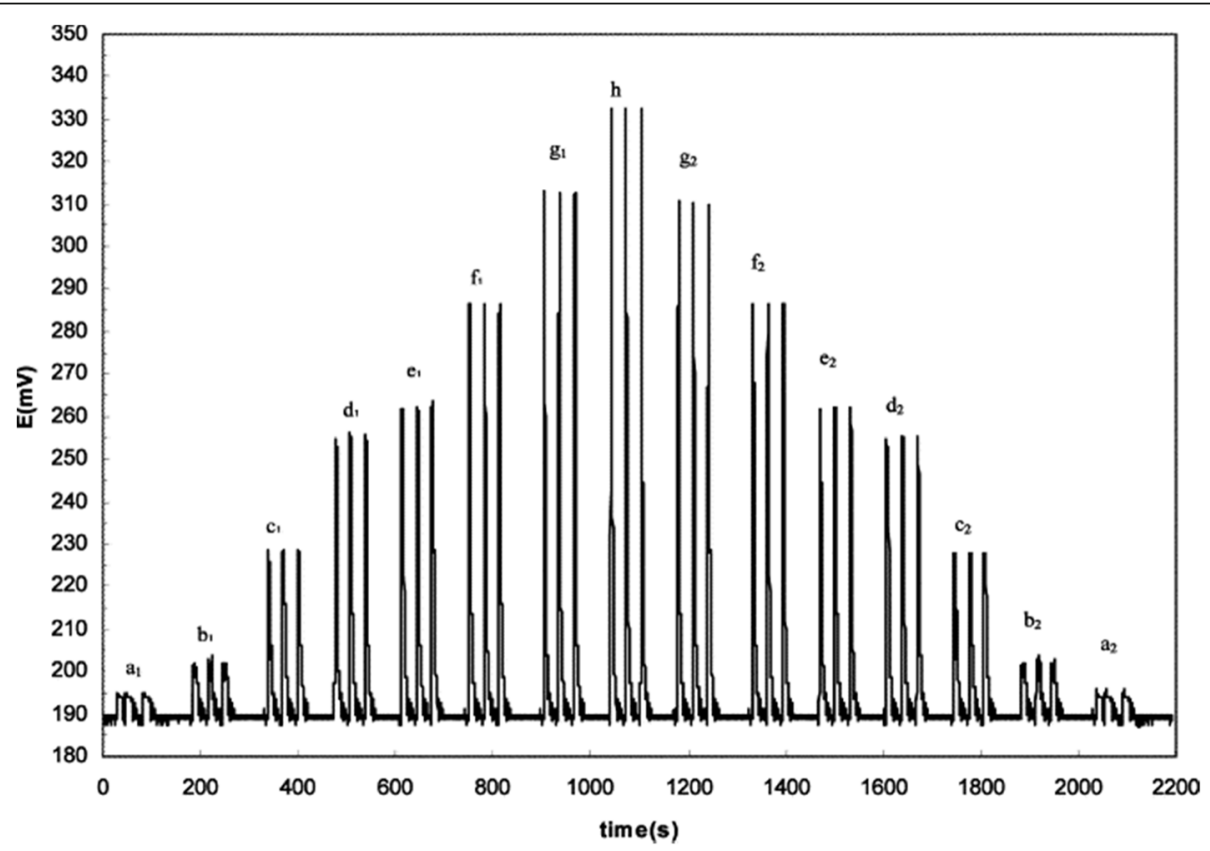

Fig. 3 Potentiometric peaks for three repetitive measurements of different $\mathrm{Pb}^{2+}$ ion concentrations. (Reprinted with permission from Reference Karami et al. 2003). (๑) 2003 Elsevier 


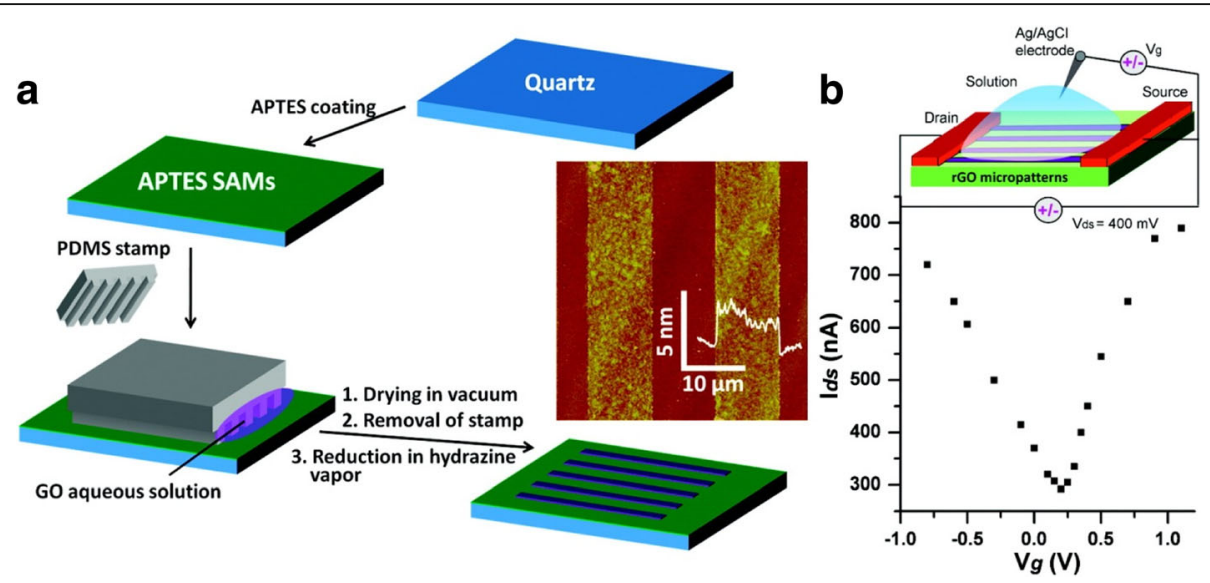

Fig. 4 a Schematic illustration for fabrication of patterned rGO thin films on APTES-coated quartz. b Ambipolar characteristics of rGO-FET measured in buffer solution. (Reprinted with permission from Reference Sudibya et al. 2011). ๑ 2011 American Chemical Society

low-cost setup, and particularly considerable electrode material availability (Fleet and Gunasinghuam 1992). Consequently, the reported electrochemical detections for environmental monitoring are mainly concentrated on potentiostatic methods, including cyclic voltammetry (CV), amperometry, and stripping voltammetry.

\section{Cyclic voltammetry}

Cyclic voltammetry is one of the most used techniques in electroanalytical studies (Rusling and Suib 1994). It provides fruitful insight into both half reactions occurring at the working electrode and the chemical or physical properties coupled to the target electrochemical reaction. In cyclic voltammetry, initiating from a potential $E_{i}$, a staircase or linear potential sweep is applied to the working electrode (Jin and Yan 2015). When reaching a switching potential of $E_{f}$, the potential sweep is reversed and returns to its initial point. The data output of CV is a current-potential curve, where the main features are the peak potentials and currents at anode and cathode. It should be noted that the half peak potential (median between the cathodic and the anodic peak potentials) reflects mainly thermodynamics characteristics, and the magnitudes of the peak current reveal the kinetics during the electrochemical reaction (Fig. 5).

The information of the electrode reaction type, the number of electron transfer, and the additional coupled phenomena (adsorption and crystallization) can also be obtained in the CV curves. For example, the electron transfer numbers are calculated using the Randles-Sevcik equation for electrochemically reversible reaction (Jin et al. 2010):

$$
i_{p}=\left(2.69 \times 10^{5}\right) n^{3 / 2} A D^{1 / 2} C v^{1 / 2}
$$

where $A$ is the surface area of the working electrode, $n$ is the number of transferred electrons, $F$ is the Faraday constant, $i_{p}$ is the peak current density, $C$ is the solubility of the oxygen, $D$ is the diffusion coefficient of the oxygen, and $v$ is the scan rate of the CV curves. As shown in Fig. 6 (Ma and Chen 2015), at grapheme-doped gold nanoparticles
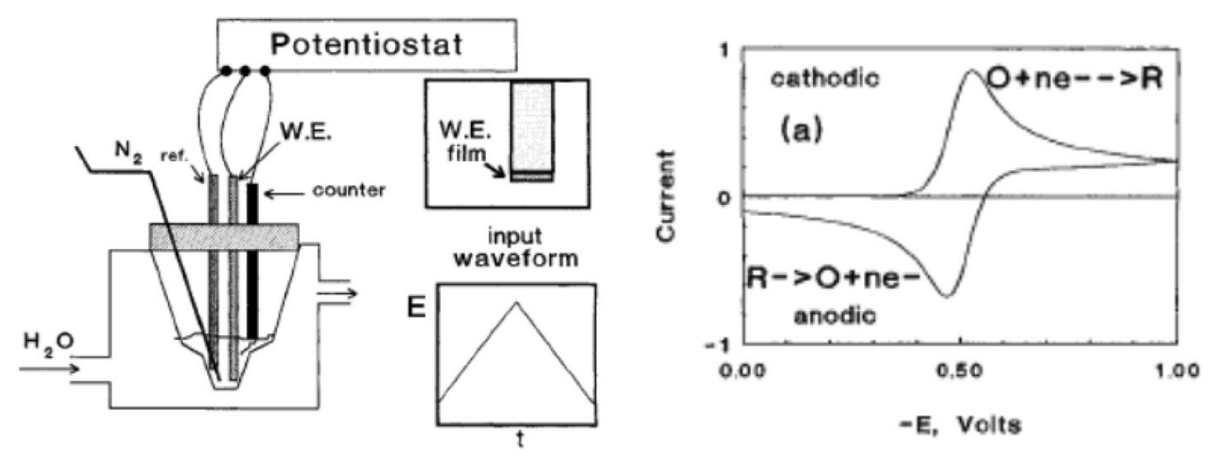

Fig. 5 (Left) A three-electrode system to measure cyclic voltammetric waveform. (Right) Ideal shape for cyclic voltammograms of a reversible diffusion-controlled reaction. (Reprinted with permission from Reference Rusling and Suib 1994). () 1994 Wiley 


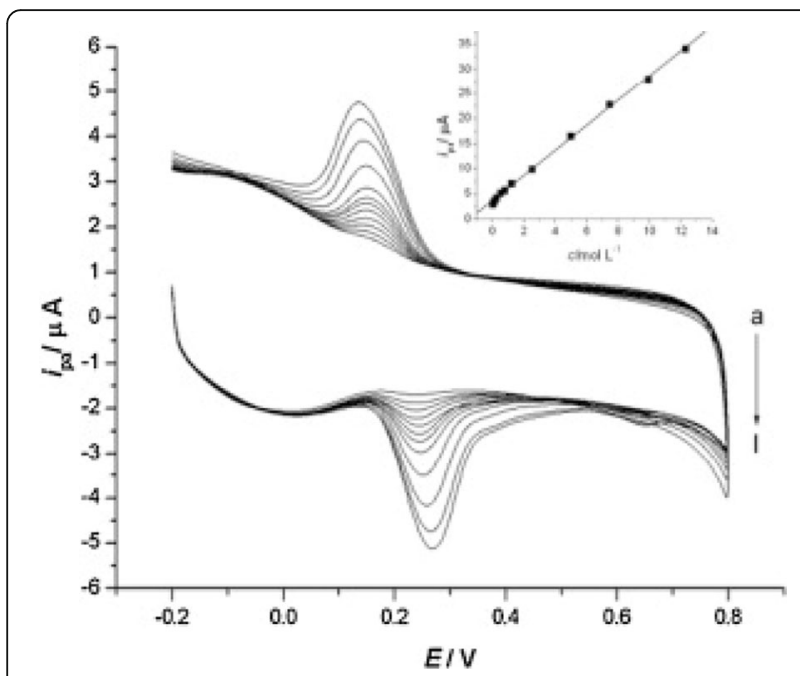

Fig. 6 CVs of DES at the GR/Nano-Au/GCE electrode with different concentrations. Inset is the plot of the oxidation peak current versus concentration of DES. (Reprinted with permission from Reference Ma and Chen 2015). (C) 2015 Elsevier

modified electrode, a good linear relationship between the oxidation peak current of CV curves, and the concentration of diethylstilboestrol (DES) was obtained from $1.20 \times 10^{-8}$ to $1.20 \times 10^{-5} \mathrm{~mol} \mathrm{~L}^{-1}$.

Another advance of this technique is fast-scan cyclic voltammetry (FSCV) at microelectrode, offering great chemical selectivity and high sensitivity for the electroactive species (Fig. 7). Sanford (Sanford et al. 2010) reported the voltammetric measurement of $\mathrm{H}_{2} \mathrm{O}_{2}$ at single, uncoated carbon-fiber microelectrodes in the presence of a catalyst. They identified both in vitro and in brain tissue that $\mathrm{H}_{2} \mathrm{O}_{2}$ can be reliably detected in the presence of multiple common interfering species.

\section{Amperometry}

Amperometry is operated by directly stepping the potential to a desired value and then determining the current or maintaining the potential at the constant value and measuring the samples at the electrode in flow injection system (Ronkainen et al. 2010). Due to the specific oxidation or reduction potential, the as-obtained current is selectively proportional to the concentration of the electroactive species in the test sample. The fixed potential also leads to a negligible charging current and minimizes the background signal that interferes the limit of detection (Rivoira et al. 2015). As shown in Fig. 8, the electrochemical cell has a weak "zero" current in the absence of sample A, and the magnitude of this blank is usually obtained during calibration at a constant polarization voltage. With the addition of sample A, species of interest start to transport across the electrolyte and are reduced at the electrode. The current of this process increases up to a plateau and reaches the equilibrium, and this plateau current monotonically related to the concentration of the target analyte.

Besides, hydrodynamic amperometric methods can significantly improve mass transport at the electrode surface by rotating and vibrating. This flow systems exhibit superior performance in environmental monitoring a
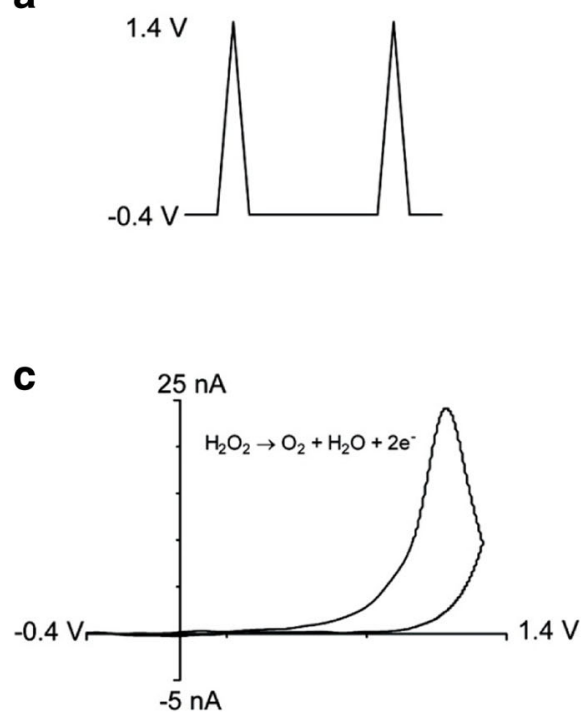

b
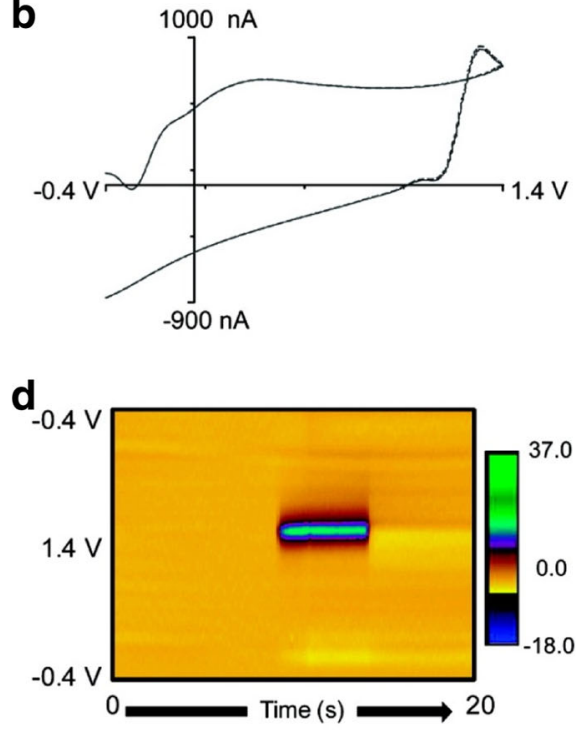

Fig. 7 Fast-scan cyclic voltammetry of $\mathrm{H}_{2} \mathrm{O}_{2}$. a The applied potential was scanned at $400 \mathrm{~V} \mathrm{~s}^{-1}$. b Background current at the carbon surface produced by the rapid scan (solid line). c Background-subtracted cyclic voltammogram of $100 \mu \mathrm{M} \mathrm{H} \mathrm{O}_{2}$ at pH 7.4. d Color plot containing 200 background-subtracted cyclic voltammograms recorded over 20 s. (Reprinted with permission from Reference Sanford et al. 2010). ( ) 2010 American Chemical Society 


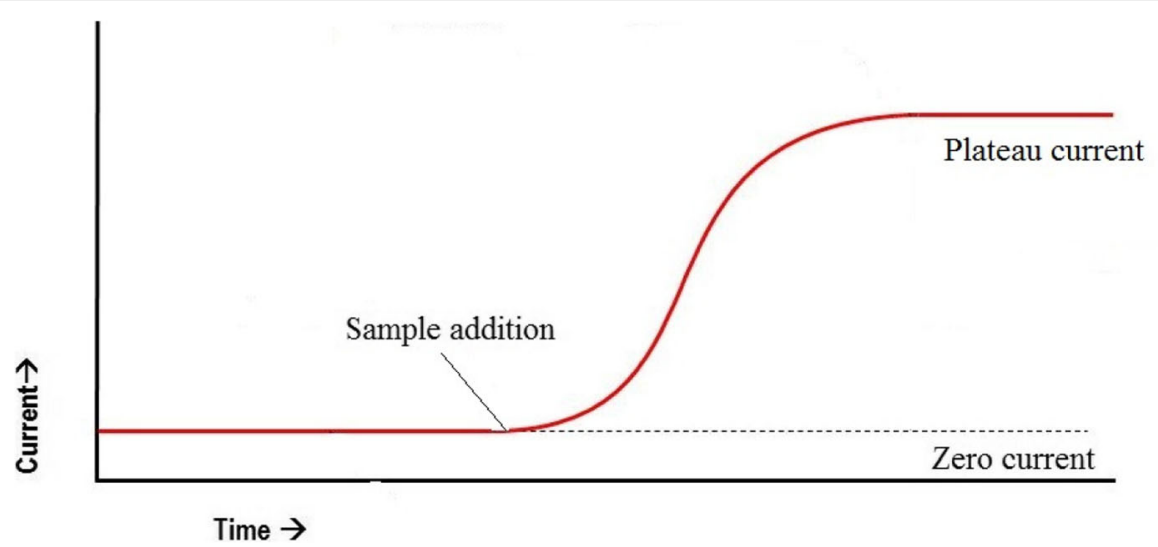

Fig. 8 Principles of amperometric measurements

than steady-state batch systems since the flow conditions allow the better solution transport in multistep assay procedures and online detection. As presented in Fig. 9 (Jin et al. 2014), the $\mathrm{Cr}(\mathrm{VI})$ concentration was detected at $\mathrm{Ti} / \mathrm{TiO}_{2} \mathrm{NT} / \mathrm{Au}$ electrode, an amperometry technique, and the potential was set at the $\mathrm{Cr}(\mathrm{VI})$ reduction peak potential. There was an excellent linear range from 0.10 to $105 \mathrm{mM} \mathrm{Cr}(\mathrm{VI})$, and more importantly, this exhibited the highest sensitivity $\left(6.91 \mu \mathrm{A} \mu \mathrm{M}^{-1}\right)$ owing to the nanostructured electrode and hydrodynamic amperometry method.

Moreover, amperometry is a widely used method for the biosensors in the environmental assessment of inorganic and organic pollutants. As illustrated in Fig. 10 (Jarque et al. 2016), the yeast biosensors are developed by coupling specific target yeast cells with electronic transducers (electrode). During the detection process, pollutant molecules enter the yeast cells and interact with biorecognition sites, such as nuclear receptors, DNA, and enzymes. As a result, an amperometric response can be detected, and the sensing information can be obtained.

\section{Stripping voltammetry}

Stripping voltammetry is a two-step method, in which the initial step consists of the electrodeposition of analyte species onto the electrode surface at a constant potential (Izadyar et al. 2016). This preconcentration step involves either anodic or cathodic process, while the second step consists of a voltage scan to obtain an electrolytic dissolution of the deposit at the electrode (stripping). Take metal ion detection for instance, the accumulation is the electrochemical reduction and deposition to metal, and then the measurement is the anodic dissolution of deposit. This process is called anodic stripping voltammetry (ASV), while the case of halides is called cathodic stripping voltammetry (CSV). The stripping step can be either linear, staircase, square wave or pulse. As shown in Fig. 11 (Baron-Jaimez et al. 2013), the three heavy metals were simultaneously detected using stripping voltammetry. However, it should be noted that the concentration at the electrode is directly determined in the stripping step rather than the concentration of an analyte in the solution. Therefore, in order
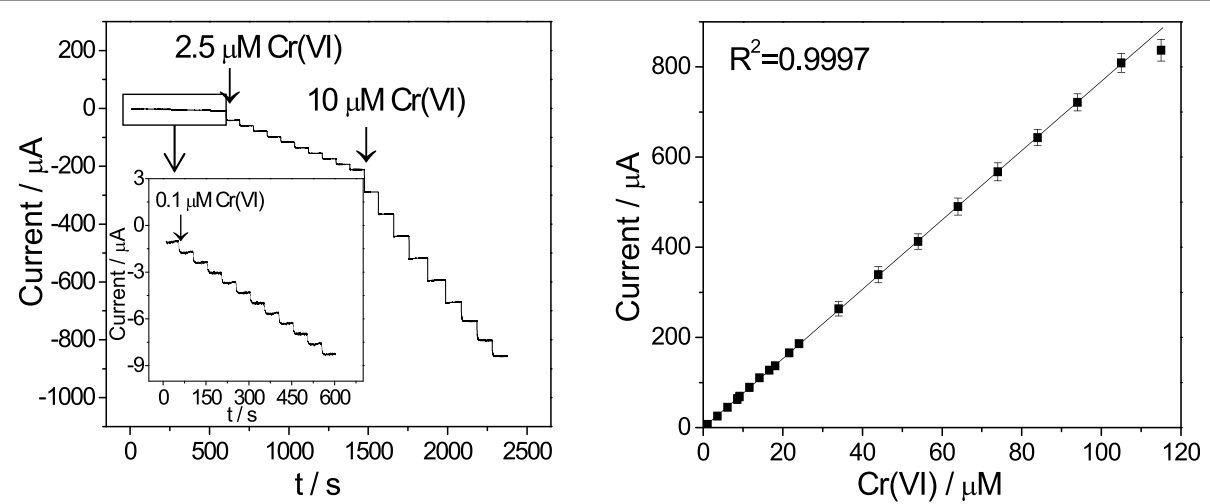

Fig. 9 (Left) Amperometric current responses of the successive addition of $\mathrm{Cr}(\mathrm{VI})$ in $0.1 \mathrm{M} \mathrm{HCl}$ solution. The inset is the enlarged part of low $\mathrm{Cr}(\mathrm{VI})$ concentration curve; (right) the calibration plot of the response against the $\mathrm{Cr}(\mathrm{VI})$ concentration (reprinted with permission from Reference Jin et al. 2014). (c) 2014 Royal Society of Chemistry 


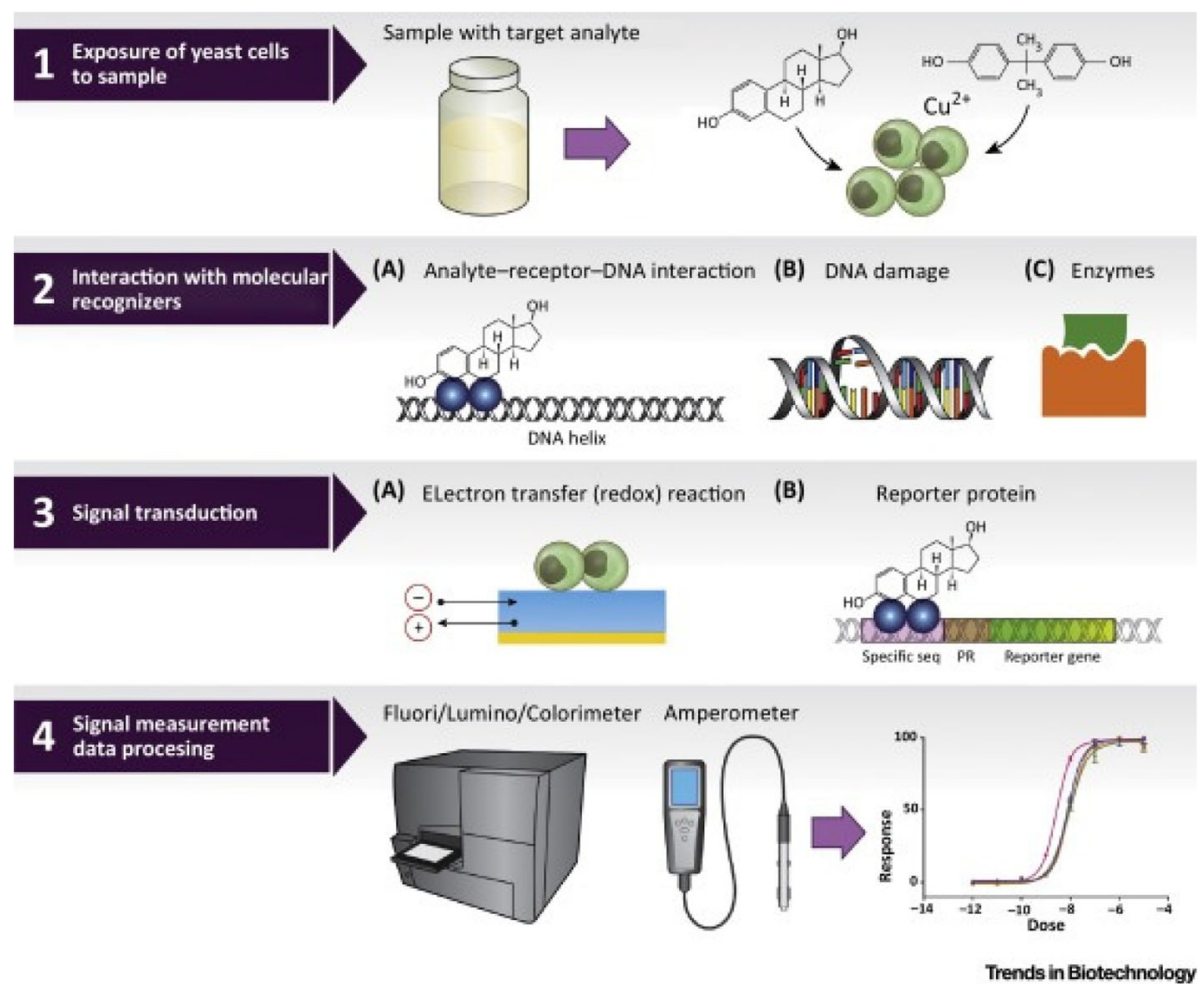

Fig. 10 Examples of immobilization strategies for yeast biosensors. (Reprinted with permission from Reference Jarque et al. 2016). ๑ 2016 Elsevier

to improve the sensitivity, the electrode concentration can be increased by increasing the deposition time and/ or the rate of stirring.

The preconcentration step of stripping voltammetry results in remarkable sensitivity particularly for the trace species at the sub-ppm level. As shown in Fig. 12 (Izadyar et al. 2016), the stripping voltammetry nearly exhibit 50-fold enhancement for the detection of citalopram at the disposable pencil lead electrode. In order to apply this sensitive method to those species that cannot be accumulated via electrolysis at the electrode surface, alternative principles have been developed with the utilization of the adsorption technique. In adsorptive stripping voltammetry (AdSV), the target analyte can be preconcentrated by physical adsorption instead of electrolytic deposition. Many organic pollutants and heavy metal cations have
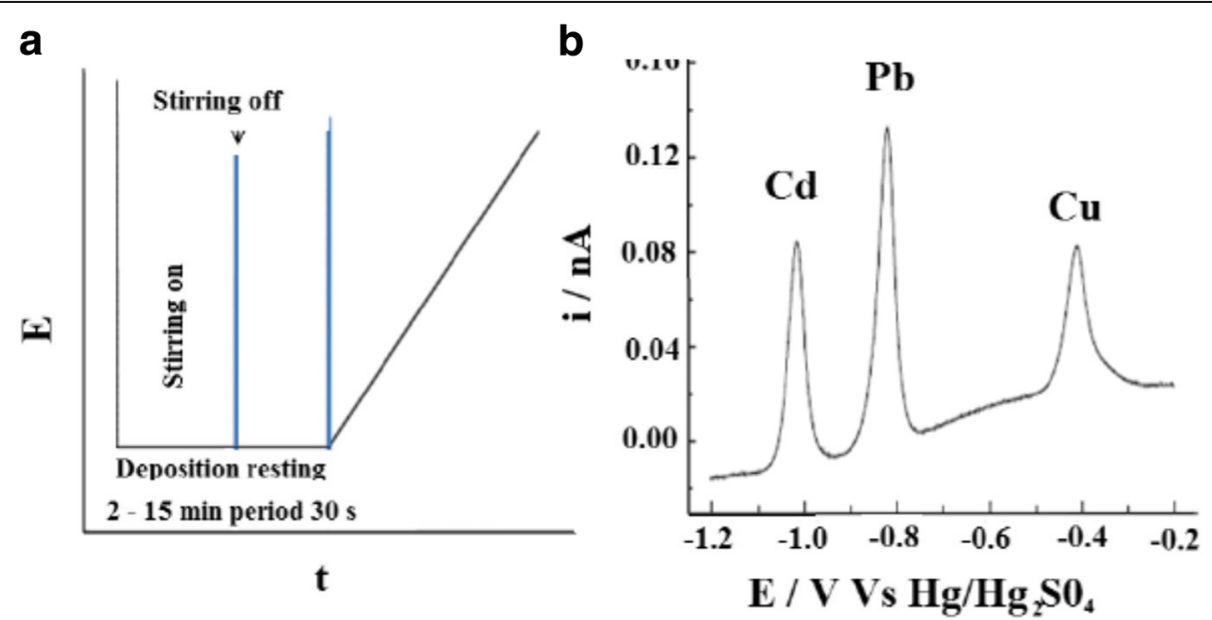

Fig. 11 a Excitation signal. b Response curve of anodic stripping voltammetry. (Reprinted with permission from Reference Baron-Jaimez et al. 2013). (๑) 2013 IOP Publishing 
a

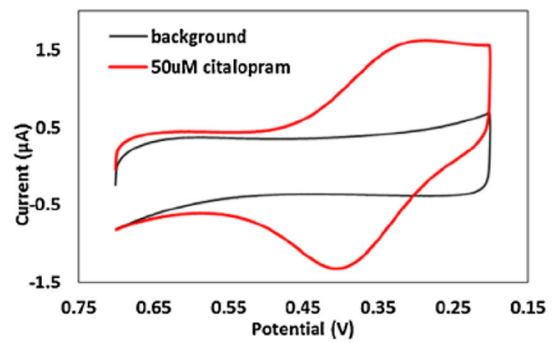

b

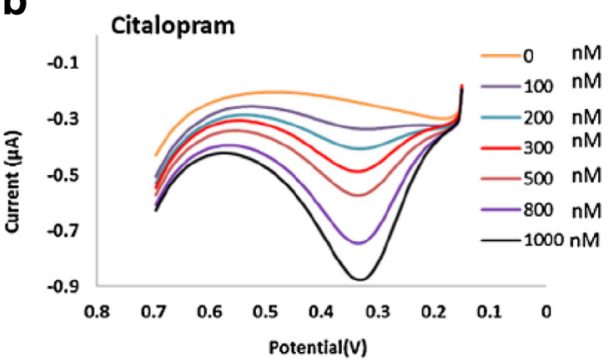

Fig. 12 (Left) CVs of 0 and $50 \mu \mathrm{M}$ citalopram and (right) stripping voltammetry of citalopram ranging from 0 to $1000 \mathrm{nM}$. (Reprinted with permission from Reference Izadyar et al. 2016). (c) 2016 Elsevier

strong affinity to be adsorbed from aqueous solution onto a mercury electrode surface, resulting in the considerable application of AdSV technique using surface-active complex agents.

\section{Impedance techniques}

Electrochemical impedance spectroscopy (EIS) (Ronkainen et al. 2010) is proposed by Lorenz and Schulze in 1975. It measures the resistive and capacitive properties of electrode upon perturbation with a small amplitude $\mathrm{AC}$ excitation, and the frequency is varied over a wide range to generate the impedance spectrum. Then, the in-phase and out-of-phase current responses are measured to determine the resistive and capacitive components of impedance, respectively. In EIS, electrochemical reactions that occur in an electrolytic cell are illustrated in terms of the electrical equivalent circuit (EEC). An idealized electrical equivalent circuit included is presented in Fig. 13 (Bansod et al. 2017), while the high- and low-frequency components are shown on the left and right, respectively. By measuring this impedance data and other resistive-capacitive (RC) characteristics of the EEC, one could determine the analyte concentration in the test sample.

EIS is a powerful detection method because they are capable of illustrating electron transfer at high frequency and mass transfer at low frequency. Impedance-based

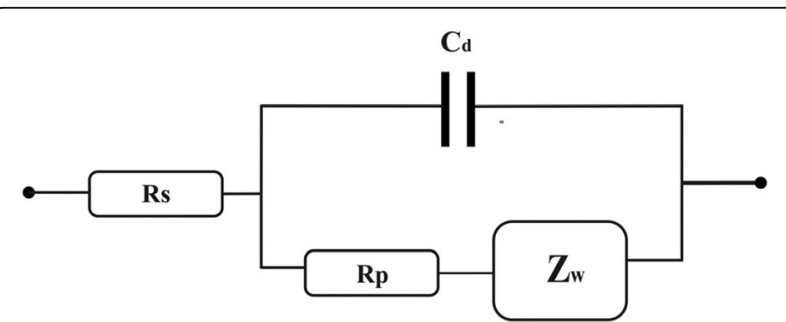

Fig. 13 An idealized Randles electrical equivalent circuit for an electrochemical reaction. $C_{\mathrm{d}}$, double-layer capacitor; $R_{\mathrm{p}}$, polarization resistance; $R_{\mathrm{s}}$, solution resistance; $Z_{W}$, Warburg impedance. (Reprinted with permission from Reference Bansod et al. 2017). (C) 2017 Elsevier detection has also been proved to be an efficient tool for the recognition of appropriate interface properties in biosensing system. It has some advantages over the widely used amperometric method since the redox mediators improve the accessibility and overcome proximity limitations to the electrode surface. As shown in Fig. 14 (Fan et al. 2013), the aptasensor concentration was determined by measuring the changes of $R_{\mathrm{ct}}$ after incubating the probe in different concentrations of acetamiprid solution for $40 \mathrm{~min}$. In the Nyquist plots of the response, the impedance obviously increases with increasing concentration of acetamiprid, indicating the increase of the electron transfer resistance. The dependence of $R_{\mathrm{ct}}$ and acetamiprid concentration is given. A linear relationship between $\Delta$ and the logarithm value of acetamiprid concentrations were obtained in the range of 5-600 $\mathrm{nM}$, indicating the facilitating effect of the redox mediator.

\section{Conductometry}

Conductometry is to monitor the changes in the electrical conductivity of the test sample (Jaffrezic-Renault and Dzyadevych 2008). This method is of great importance in the detection of toxic, harmful gases, like $\mathrm{SO}_{2}$, $\mathrm{NO}_{\mathrm{x}}, \mathrm{H}_{2} \mathrm{~S}, \mathrm{CO}$, and $\mathrm{NH}_{3}$. Conductometric (resistive) gas sensors are widely used for the environmental monitoring; these "solid-state" sensors possess an excellent sensitivity, short response time, low cost, and device feasibility. The detection mechanism originates from a change in conductivity via trapping of electrons and band bending induced by the adsorbed analyte molecules. As shown in Fig. 15 (Shu et al. 2010), the course of the measured resistance $R$ during cyclic exposure to $\mathrm{NO}_{2}$ at iron (II) phthalocyanine is presented. While dosing $\mathrm{NO}_{2}$ in $\mathrm{N}_{2}$ with a fixed concentration of $0.5,1$, and $2 \mathrm{ppm}$, the resistance decreases with a steady slope and then remains at the corresponding level during the intermediate $\mathrm{N}_{2}$ phases, leading to plateaus in the detection course.

Conductometry has also been employed in biosensors for environmental monitoring and clinical analysis. As illustrated in Fig. 16 (Anh et al. 2004), the conductometric 

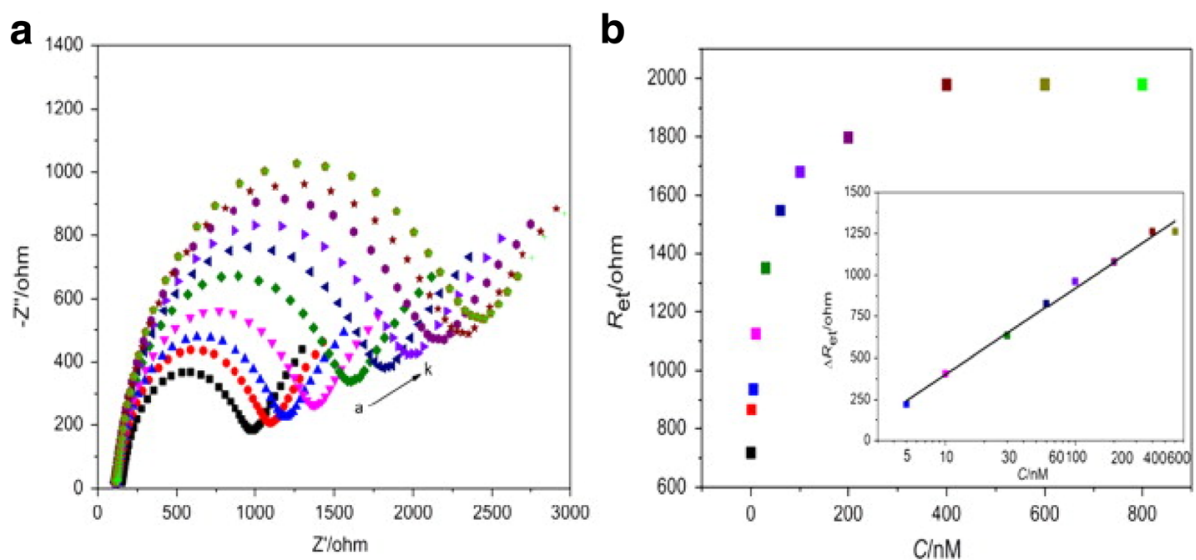

Fig. 14 a Nyquist plots of the MCH/aptamer/AuNPs/gold electrode correspond to different concentrations of acetamiprid. $\mathbf{b}$ The dependence of $R_{\text {et }}$ of on the concentration of acetamiprid; inset is the linear calibration curve of $\Delta R_{\text {et }}$ with the logarithm of acetamiprid concentrations (reprinted with permission from Reference Fan et al. 2013). @ 2013 Elsevier

transducer is a miniature two-electrode device fabricated to determine the conductivity of the thin electrolyte layer between the electrode surfaces. Two pairs of Pt-integrated electrodes (150 nm thick) were developed on the Pyrex glass substrate $(10 \mathrm{~mm} \times 30 \mathrm{~mm})$; tyrosinase was immobilized on the biosensor detection part. As a result, the concentration of diuron, atrazine, desisopropylatrazine (DIA) and desethylatrazine (DEA) was successfully detected at the "sensitive" electrode.

\section{Electrochemiluminescence techniques}

Electrochemiluminescence (ECL, called electrogenerated chemiluminescence) involves the formation of species at electrode surfaces which then proceed to electron-transfer reactions to generate an electronically excited state that emits light (Richter 2004). Electrochemiluminescence from

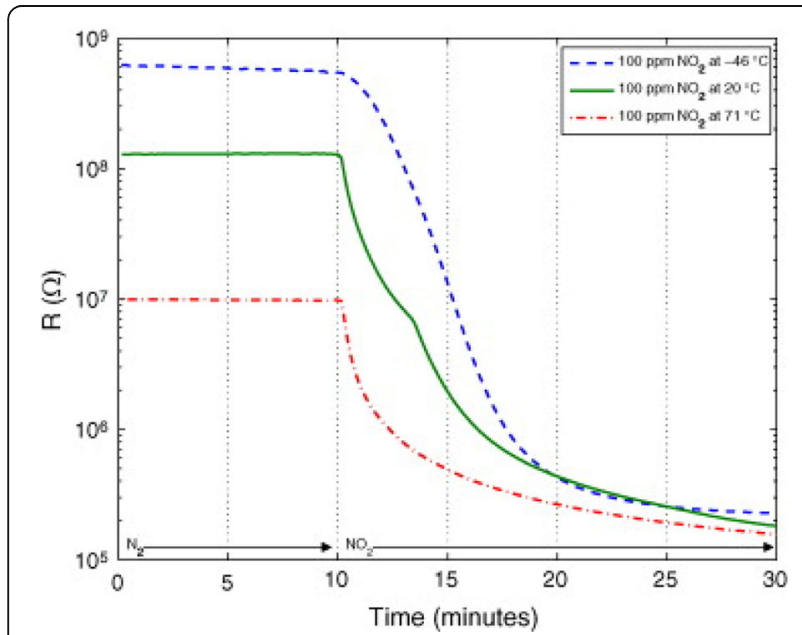

Fig. 15 Response of sensors to 30-min $100 \mathrm{ppm} \mathrm{NO}$ exposure (reprinted with permission from Reference Shu et al. 2010). ( 2010 Elsevier
$\mathrm{Ru}(\mathrm{bpy})_{3}{ }^{2+}$ was first developed in 1972 in acetonitrile $(\mathrm{MeCN})$ using tetrabutylammonium tetrafluoroborate $\left(\mathrm{TBABF}_{4}\right)$ as the solvent. ECL was formed by change pulsing of an electrode potential to form oxidized $\mathrm{Ru}$-(bpy) ${ }_{3}{ }^{3+}$ and reduced $\mathrm{Ru}(\mathrm{bpy})_{3}{ }^{+}$(Fig. 17). Therefore, ECL is observed during the application of potential (several volts) to electrode materials in the aprotic organic solvent of luminescent species, which are usually polycyclic aromatic hydrocarbons, metal complexes, quantum dots, or nanoparticles. It should be noted that simultaneous redox reactions of luminescent species are difficult to achieve in an aqueous medium due to the electrochemical water splitting.

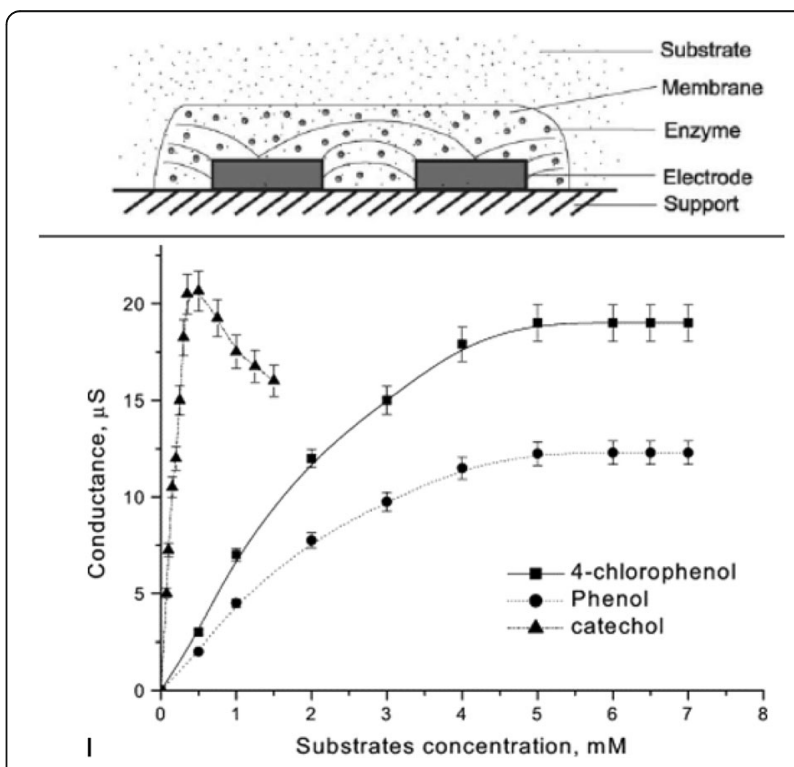

Fig. 16 (Upper) Schematic representation of conductometric biosensor; (below) calibration curves of conductometric tyrosinase biosensor for 4-chlorophenol, phenol, and catechol. (Reprinted with permission from Reference Anh et al. 2004). ( 2004 Elsevier 


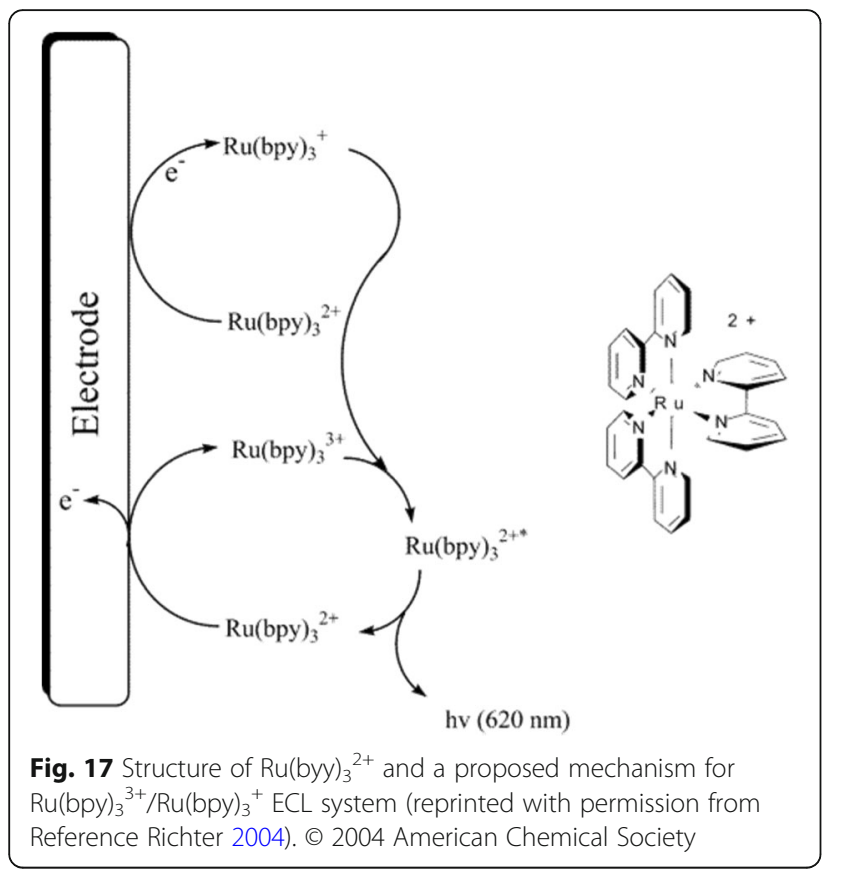

ECL is a highly sensitive and selective analytical method. It combines both advantages of chemiluminescent (elimination of background optical signal) and ease process control by applying electrode potential. Recently, a novel electrochemiluminescence (ECL) sensor based on carbon quantum dots (CQDs) immobilized on graphene (GR) has been reported for the detection of chlorinated phenols (CPs) in water as presented in Fig. 18 (Yang et al. 2013). The low ECL intensity of CQDs in the aqueous system has been overcome through multistage amplification of the CQD ECL signal by GR and $\mathrm{S}_{2} \mathrm{O}_{8}{ }^{2-}$. The as-prepared
ECL sensor enables the real-time monitoring of PCP with an unprecedented sensitivity of $1.0 \times 10^{-12} \mathrm{M}$ and a wide linear range from $1.0 \times 10^{-12}$ to $1.0 \times 10^{-8} \mathrm{M}$.

\section{Conclusions}

This minireview has described to emphasize the electroanalytical techniques employed for the sensing of environmental pollutants based on nanomaterials as the exceptional analytical tools among other analytical methods in use today. Electroanalytical chemistry offers considerable promise for the environmental sensing platforms, with features of remarkable sensitivity and selectivity, low detection limit, inherent miniaturization, and portability. The analytes can be electrochemically monitored with a measurable potential (potentiometry), current (cyclic voltammetry, amperometry, stripping voltammetry), impedance (EIS), and conductivity (conductometry) as well as the recently updated electrochemiluminescence (ECL). With respect to numerous pollutant systems, various electroanalytical techniques should be carefully selected to obtain better sensing performance. The sensitivity and selectivity of the environmental sensor platforms have always been coupled with their selection of electroanalytical tools, electrode materials of nanomaterials, and electrolytes. The applications of electroanalytical methods have been evidenced for numerous applications in diagnostics, biological, biomedical, environmental, and food industries. The advancement of novel analytical methods with an excellent electrochemical active electrode surfaces based on functional nanomaterials offers the prospect of environmental sensors that will display even rapid response and high sensitivity. With the knowledge of electrochemistry and a better indulgent of

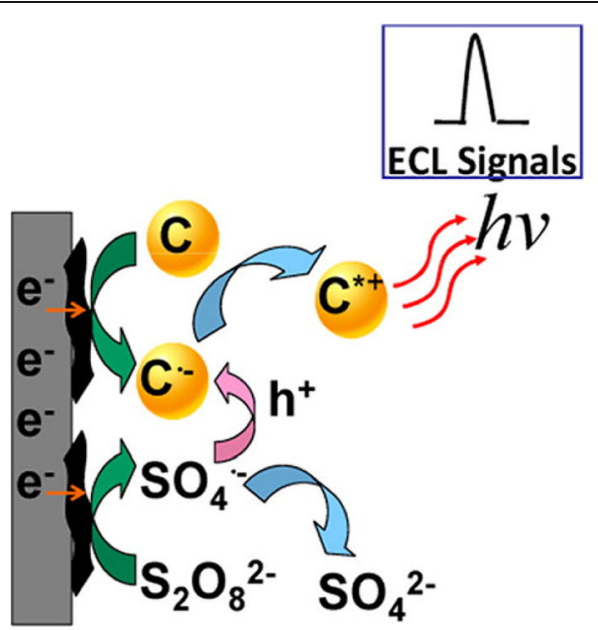

ECL emission

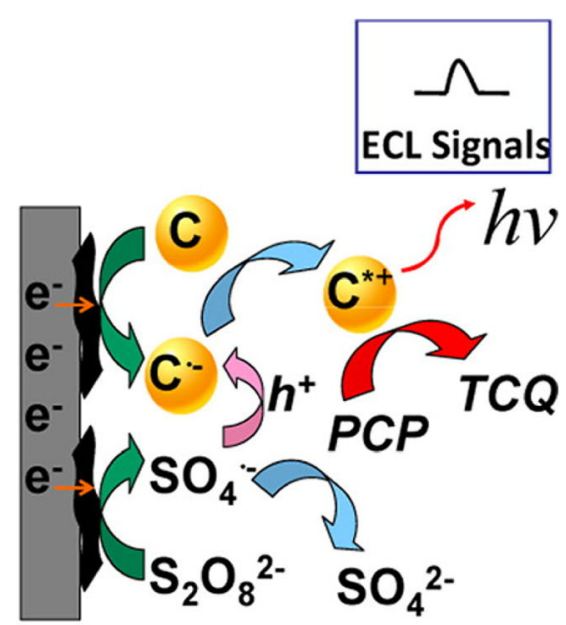

ECL quenching

Fig. 18 Illustrative ECL detection mechanism for PCP with CQD/GR in $\mathrm{S}_{2} \mathrm{O}_{8}{ }^{2-}$ solution (reprinted with permission from Reference Yang et al. 2013). (c) 2013 American Chemical Society 
electrode fabrication with micro- and nano-scale electrodes and testing, the performance of sensor platforms will improve considerably in terms of both sensitivity and detection limits. Electroanalytical techniques will certainly participate a significant role in their development, and the advancement in these areas will be persuaded to spark innovations in health, industry, and society as an intact.

\section{Acknowledgements}

WJ acknowledges the funding support from the National Natural Science Foundation of China under Grant No. 51604253 and "CAS Pioneer Hundred Talents Program".

Availability of data and materials

Data are available on request.

\section{Authors' contributions}

This review was primarily designed and written by WJ and GM. Both authors read and approved the final manuscript.

\section{Competing interests}

Both authors declare that they have no competing interests.

\section{Publisher's Note}

Springer Nature remains neutral with regard to jurisdictional claims in published maps and institutional affiliations.

Received: 30 May 2018 Accepted: 2 August 2018

Published online: 31 August 2018

\section{References}

Anh TM, Dzyadevych SV, Van MC, Renault NJ, Duc CN, Chovelon J-M. Conductometric tyrosinase biosensor for the detection of diuron, atrazine and its main metabolites. Talanta. 2004;63:365.

Bansod BK, Kumar T, Thakur R, Rana S, Singh I. A review on various electrochemical techniques for heavy metal ions detection with different sensing platforms. Biosensors and Bioelectronics. 2017;94:443-55.

Baron-Jaimez J, Joya MR, Barba-Ortega J. Anodic stripping voltammetry-ASV for determination of heavy metals. J Phys-Concend Mat. 2013;446:012023.

Chin Y-L, Chou J-C, Sun T-P, Chung W-Y, Hsiung S-K. A novel pH sensitive ISFET with on chip temperature sensing using CMOS standard process. Sensor Actuat B-Chem. 2001;76:582.

Crespo GA. Recent advances in ion-selective membrane electrodes for in situ environmental water analysis. Electrochim Acta. 2017:245:1023.

Dimeski G, Badrick T, John AS. Ion Selective Electrodes (ISEs) and interferences--a review. Clin Chem Acta. 2010:411:309.

Fan L, Zhao G, Shi H, Liu M, Li Z. A highly selective electrochemical impedance spectroscopy-based aptasensor for sensitive detection of acetamiprid. Biosens Bioelectron. 2013;43:12.

Fleet B, Gunasinghuam $\mathrm{H}$. Electrochemical sensors for monitoring environmental pollutants. Talanta. 1992;39:1449.

Izadyar A, Arachchige DR, Cornwell H, Hershberger JC. Ion transfer stripping voltammetry for the detection of nanomolar levels of fluoxetine, citalopram, and sertraline in tap and river water samples. Sens Actuat B-Chem. 2016;223: 226.

Jaffrezic-Renault N, Dzyadevych SV. Conductometric Microbiosensors for Environmental Monitoring. Sensors. 2008:8:2569.

Jarque S, Bittner M, Blah L, Hilscherov K. east Biosensors for Detection of Environmental Pollutants: Current State and Limitations. Trends Biotechnol. 2016;34:408.

Jin W, Du H, Zheng $\mathrm{S}, \mathrm{Xu} \mathrm{H}$, Zhang Y. Comparison of the oxygen reduction reaction between $\mathrm{NaOH}$ and $\mathrm{KOH}$ solutions on a Pt electrode: The electrolyte-dependent effect. J Phys Chem B. 2010;114:6542.

Jin W, Du H, Zheng S, Zhang Y. Electrochemical processes for the environmental remediation of toxic Cr(VI): A review. Electrochim Acta. 2016;191:1044.

Jin W. Maduraiveeran G. lectrochemical detection of chemical pollutants based on gold nanomaterials. Trends Environ Anal Chem. 2017;14:28.
Jin W, Wu G, Chen A. Sensitive and selective electrochemical detection of chromium(VI) based on gold nanoparticle-decorated titania nanotube arrays. Analyst. 2014;139:235

Jin W, Yan K. Recent advances in electrochemical detection of toxic $\mathrm{Cr}(\mathrm{VI})$. RSC Adv. 2015;5:37440.

Karami H, Mousavi MF, Shamsipur M. Flow injection potentiometry by a new coated graphite ion-selective electrode for the determination of $\mathrm{Pb} 2+$ Talanta. 2003;60:775.

Li T, Wu Y, Huang J, Zhang S. Gas sensors based on membrane diffusion for environmental monitoring. Sens Actuat B-Chem. 2017;243:566.

Ma X, Chen M. lectrochemical sensor based on graphene doped gold nanoparticles modified electrode for detection of diethylstilboestrol. Sensor Actuat B-Chem. 2015;215:445.

Maduraiveeran G, Jin W. Nanomaterials based electrochemical sensor and biosensor platforms for environmental applications. Trends Environ Anal Chem. 2017;13:10.

Matzeu G, Zuliani C, Diamond D. Solid-contact ion-selective electrodes (ISEs) based on ligand functionalised gold nanoparticles. Electrochim Acta. 2015; 159:158.

Qin Y, Peper S, Bakker E. Plasticizer-free polymer membrane ion-selective electrodes containing a methacrylic copolymer matrix. Electroanalysis. 2002; 14:1375

Ricci F, Volpe G, Micheli L, Palleschi G. A review on novel developments and applications of immunosensors in food analysis. Anal Chim Acta. 2007;605:111.

Richter MM. Electrochemiluminescence (ECL). Chem Rev. 2004;104:3003.

Rivoira L, De Carlo RM, Cavalli S, Bruzzoniti MC. Simple SPE-HPLC determination of some common drugs and herbicides of environmental concern by pulsed amperometry. Talanta. 2015;131:205.

Ronkainen NJ, Halsall HB, Heineman WR. Electrochemical biosensors. Chem Soc Rev. 2010:39:1747.

Rusling JF, Suib SL. Electroluminescence from oligothiophene-based lightemitting devices. Adv Mater. 1994:12:922.

Sanford AL, Morton SW, Whitehouse KL, Oara HM, Lugo-Morales LZ, Roberts JG, Sombers LA. Voltammetric detection of hydrogen peroxide at carbon fiber microelectrodes. Anal Chem. 2010;82:5205.

Shao Y, Wang J, Wu H, Liu J, Aksay IA, Lin Y. Graphene based electrochemical sensors and biosensors: A review. Electroanalysis. 2010;22:1027.

Shu JH, Wike HC, Chin BA. Passive chemiresistor sensor based on iron (II) phthalocyanine thin films for monitoring of nitrogen dioxide. Sens Actuat BChem. 2010;148:498.

Sudibya HG, He Q, Zhang H, Chen P. Electrical detection of metal ions using field-effect transistors based on micropatterned reduced graphene oxide films. ACS Nano. 2011:5:1990.

Wang J. Electrochemical detection for microscale analytical systems: a review. Talanta. 2002;56:223.

Yang S, Liang J, Luo S, Liu C, Tang Y. Supersensitive detection of chlorinated phenols by multiple amplification electrochemiluminescence sensing based on carbon quantum dots/graphene. Anal Chem. 2013;85:7720.

Zuliani C, Diamond D. Opportunities and challenges of using ion-selective electrodes in environmental monitoring and wearable sensors. Electrochim Acta. 2012;84:29.

\section{Submit your manuscript to a SpringerOpen ${ }^{\circ}$ journal and benefit from:}

- Convenient online submission

- Rigorous peer review

- Open access: articles freely available online

High visibility within the field

- Retaining the copyright to your article

Submit your next manuscript at $>$ springeropen.com 